\title{
A Study of the Thermal Transition of Poly(2-vinylpyridine) in Solution by Carbon-13 NMR
}

\author{
Yoshio Inoue, Tetsuo Konno, and Katsumi NaKajima \\ Department of Polymer Chemistry, Tokyo Institute of Technology, \\ 12-1, Ookayama 2-chome, Meguro-ku, Tokyo 152, Japan.
}

(Received October 5, 1976)

\begin{abstract}
The variation, as a function of temperatute, of the carbon-13 spinlattice relaxation time of individual carbons of poly(2-vinylpyridine) in solution was measured and the molecular motion was analysed quantitatively. The motion of the polymer can be characterized by three contributions: namely, a segmental motion and two kinds of internal rotation of the pyridine ring. The temperature dependence of the rate of the internal rotation revealed a transitional change at about $25^{\circ} \mathrm{C}$, while that of the segmental motion changed linearly. It is suggested that the internal rotation of the pyridine ring is interrupted by steric hindrance, which is probably due to a repulsive interaction between the hydrogen atoms in the pyridine ring and the methine group and by a formation of hydrogen bonding at lower temperatures. Above the transition temperature, the thermally activated motion overcomes such hindrances and the pyridine ring rotates more freely.

KEY WORDS Poly(2-vinylpyridine) / ${ }^{13} \mathrm{C}-\mathrm{NMR} /$ Spin-Lattice Relaxation / Molecular Motion / Internal Rotation / Transition /
\end{abstract}

Recently much interest has been devoted by several investigators to transitions observed in the temperature dependence of the conformation of a macromolecular chain. It has been generally accepted that solutions of polystyrene show an anomalous temperature dependence in solution properties at about 50 and $80^{\circ} \mathrm{C}^{1-3} \mathrm{~A}$ transition was observed at $80^{\circ} \mathrm{C}$ for both atactic and isotactic polystyrenes in several solvents in the temperature dependence of the second virial coefficient, in the mean radius of gyration, and so on. Reiss ${ }^{4,5}$ has proposed that this arises from the transition between a $3_{1}$ helix and a random coil in the isotactic sequences of both polymers. On the other hand, a transition at about $50^{\circ} \mathrm{C}$ for isotactic and atactic polystyrenes both in solution and bulk has been reported. ${ }^{1}$ In a previous paper, ${ }^{6}$ this phenomenon was investigated by using carbon-13 nuclear-magneticresonance spectroscopy (NMR) and it was concluded that this transition is due to the sudden modal change in the mobility of the phenyl ring.
Several properties, such as viscosity ${ }^{7}$ and optical density, ${ }^{8}$ of poly(2-vinylpyridine) in solution have also been investigated, and a transition was observed at about $25^{\circ} \mathrm{C}$ in several solvents in the temperature dependence of these properties. Chachaty, et al., ${ }^{9}$ have investigated the temperature dependence of the carbon-13 spin-lattice relaxation time of backbone methine carbon of isotactic poly(2-vinylpyridine); but they found no anomaly because the temperature range of their measurement $\left(30-80^{\circ} \mathrm{C}\right)$ was above the transition temperature $\left(25^{\circ} \mathrm{C}\right)$, and they observed only linearity in the temperature dependence of the correlation time. The process of the transition is different according to the kind of solvents. A break point has been observed in the temperature dependence of the intrinsic viscosity of poly(2-vinylpyridine) in benzene and tetrahydrofuran solution, but no break point has been observed in chloroform. ${ }^{8}$ In this paper we will present the results of the measurement of the carbon-13 magnetic relaxation time and ultraviolet-absorption (UV) spectrum of poly(2-viny- 
pyridine) in the temperature range from 15 to $75^{\circ} \mathrm{C}$. We intend to analyse the transition mechanism in the vicinity of $25^{\circ} \mathrm{C}$ of poly (2vinypyridine) in a solution of benzene and chloroform.

\section{EXPERIMENTAL}

\section{Materials}

According to the method of Natta, et al., ${ }^{10}$ isotactic poly(2-vinylpyridine) was prepared by anionic polymerization in toluene using phenyl magnesiumbromide as a catalyst. The intrinsic viscosity number in benzene solution at $25^{\circ} \mathrm{C}$ was measured, and by use of the equation ${ }^{11}$

$$
[\eta]=1.70 \times 10^{-4} M_{v}
$$

the viscosity-averaged molecular weight $\left(M_{v}\right)$ was estimated to be 55100. The solvents used were benzene- $d_{6}\left(\mathrm{C}_{6} \mathrm{D}_{6}\right)$ as a nonpolar solvent, and chloroform- $d\left(\mathrm{CDCl}_{3}\right)$ as a polar solvent; these were purchased from Commissariat a l'Energie Atomique (France).

\section{Methods}

Ultraviolet-absorption spectra were observed on a Beckman-25 spectrometer at several temperatures.

Carbon-13 spectra were measured using a JEOL PS-100 spectrometer equipped with a PFT100 Fourier transform system operating at 25.15 $\mathrm{MHz}$ and a magnetic drum with $8-\mathrm{K}$ words (1 word $=16$ bits) as an auxiliary memory device; this system yielded 4096 data points in the Fourier transformed spectrum. The $90^{\circ}$ pulse width was about $20 \mu \mathrm{sec}$.
The apparatus was equipped with an internal ${ }^{2} \mathrm{D}$ lock and a noise modulated proton decoupler. The $90^{\circ}$ pulse recycle times were chosen to be at least five times the longest spin-lattice relaxation time of the carbon atoms of interest in the polymer. Samples were contained in 8-mm O.D. glass tubes and were not degassed, since the relaxation times of the polymer are not substantially affected by the presence of dissolved oxygen at least at atomospheric pressure. ${ }^{12}$ The polymer concentration was $25 \%(\mathrm{w} / \mathrm{v})$.

In Figure 1 is shown the carbon-13 NMR spectrum of isotactic poly(2-vinylpyridine) in $\mathrm{C}_{6} \mathrm{D}_{6}$ at $35^{\circ} \mathrm{C}$. The assignment of the individual peaks to the corresponding carbons in the polymer has been made by Chachaty, et al., ${ }^{9}$ as shown in Figure 1. The multiplet resonance which appears between peaks 3 and 4 is the resonance of $\mathrm{C}_{6} \mathrm{D}_{6}$.

\section{$T_{1}$ Measurement}

Spin-lattice relaxation times $\left(T_{1}\right)$ for each carbon were determined from proton decoupled partially relaxed Fourier transform (PRFT) spec$\mathrm{tra}^{13}$ obtained by means of the conventional $180^{\circ}-t-90^{\circ}$ pulse sequence, where $t$ is the time interval between the $180^{\circ}$ and the $90^{\circ}$ pulses. The intensity $M(t)$ of the peaks obtained from sets of such PRFT spectra was used to determine $T_{1}$ according to the equation

$$
M(t)=M_{0}\left[1-2 \exp \left(-t / T_{1}\right)\right]
$$

where $M_{0}$ is the equilibrium intensity measured in a normal FT spectrum. The assumption of exponential decay in eq 1 was justified by the

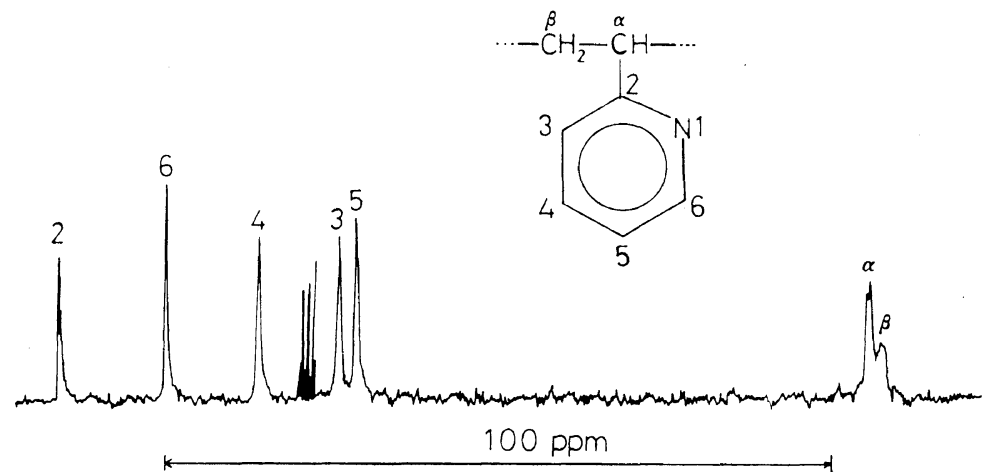

Figure 1. Proton-decoupled, natural abundance ${ }^{13} \mathrm{C}$ FT NMR spectrum of isotactic poly(2vinylpyridine) in benzene- $d_{6}$ at $25.15 \mathrm{MHz}$ and $35^{\circ} \mathrm{C}$. The spectrum is from 512 accumulations of time-domain signals with a repetition time of $1.2 \mathrm{sec}$. 
good linearity in the plots of $\log \left[M_{0}-M(t)\right] v$ s. $t$. More than three runs were carried out; the maximum error of $T_{1}$ was estimated to be within $\pm 10 \%$.

\section{RESULTS}

\section{UV Spectrum}

In Figure 2 is shown the temperature dependence of the optical density of the UV spectrum at the absorption maximum for poly(2-vinylpyridine) in chloroform. The variation of the optical density, as a function of increasing temperature, shows three parts as follows: uniform decrease (below $25^{\circ} \mathrm{C}$ ), sharp decrease $\left(25-35^{\circ} \mathrm{C}\right)$, and uniform decrease (above $35^{\circ} \mathrm{C}$ ). This figure clearly shows the occurrence of a transition between 25 and $35^{\circ} \mathrm{C}$. This is consistent with the results appearing in the literatures. ${ }^{7,8}$ The UV spectrum of poly(2-vinylpyridine) in benzene was not observed because of the overlapping of the solvent peak on the solute peak.

\section{Spin-Lattice Relaxation Time}

In Figure 3 is shown the temperature dependence of $T_{1}$ for carbons in poly(2-vinylpyridine). $T_{1}$ values for $\mathrm{C}(5)$ and main chain $\mathrm{C}(\alpha)$ carbons increase linearly with increasing temperature, while those for $C(3), C(4)$, and $C(6)$ may be constructed of two regions, namely, a straight line with a smaller slope in the lower temperature region and that with a larger slope in the higher temperature region. A similar behavior is observed in the temperature dependence of poly(2-vinylpyridine) in $\mathrm{CDCl}_{3}$. Since the peak height of the $C(\beta)$ resonance is smaller than

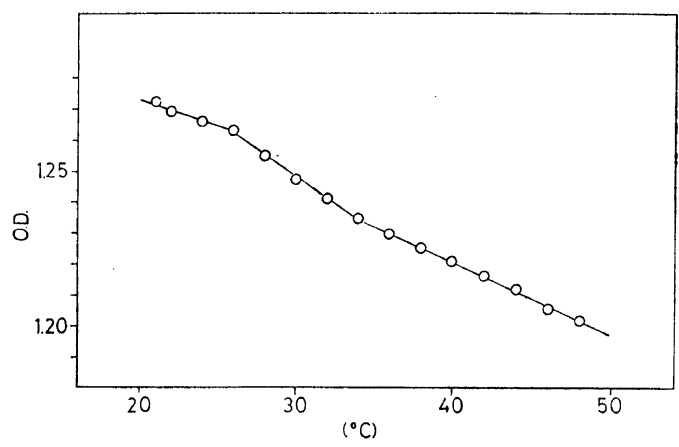

Figure 2. Temperature dependence of optical density of poly(2-vinylpyridine) in chloroform.

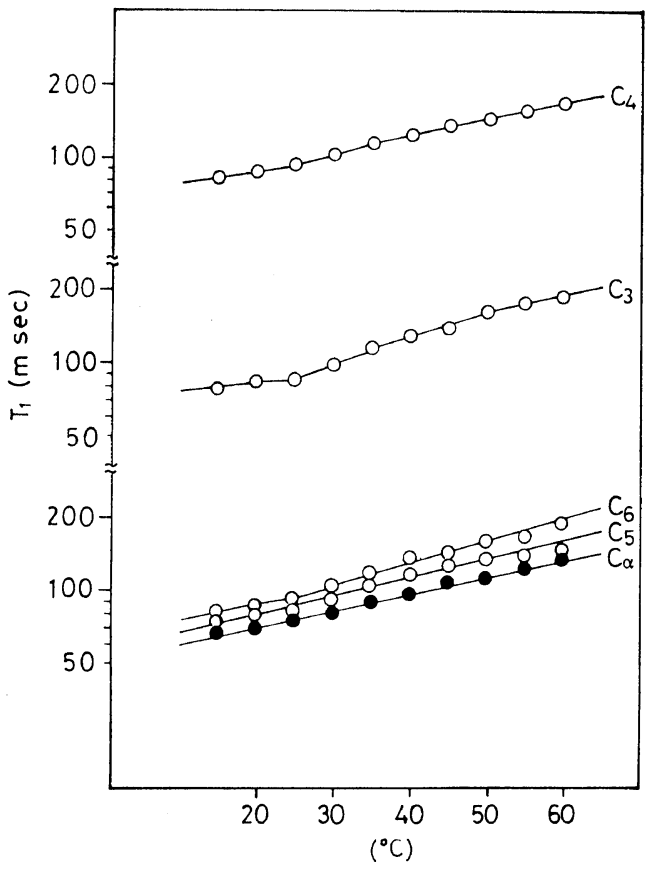

(a)

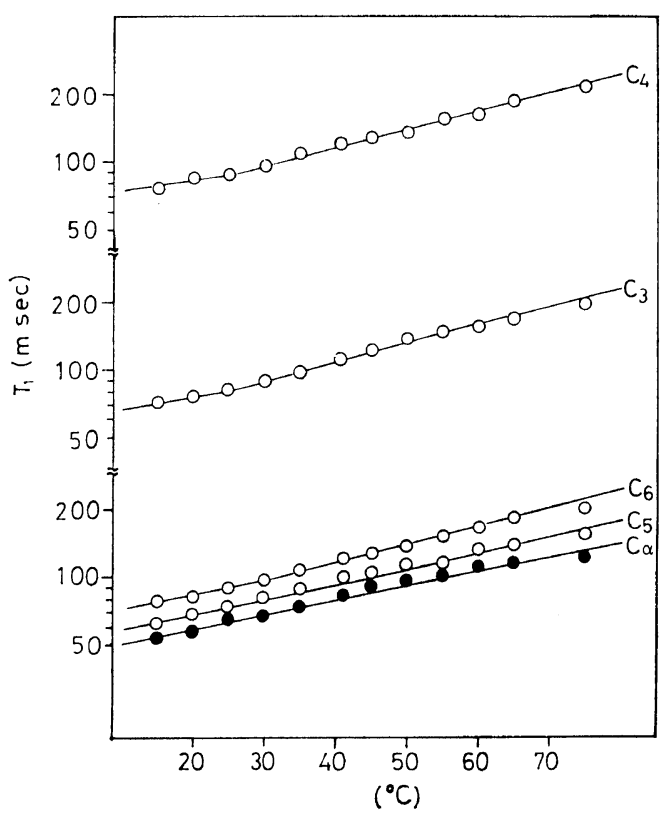

(b)

Figure 3. Temperature dependence of the spinlattice relaxation time $\left(T_{1}\right)$ : (a) poly(2-vinylpyridine) in chloroform-d; (b) poly(2-vinylpyridine) in benzene- $d_{6}$. 
those of the other carbons, as shown in Figure 1 , the accurate measurement of $T_{1}$ for $\mathrm{C}(\beta)$ is difficult, and so the values of $T_{1}$ are not shown in Figure 3.

\section{Correlation Time}

If the ${ }^{13} \mathrm{C}-{ }^{1} \mathrm{H}$ dipolar interaction is predominant in the carbon-13 magnetic relaxation mechanism and the segmental motion is isotropic, $T_{1}$ is given by

$$
\begin{aligned}
\frac{1}{T_{1}}= & \frac{\hbar}{10} \gamma_{\mathrm{c}}{ }^{2} \gamma_{\mathrm{H}}{ }^{2} \sum r_{i}^{-6}\left[f\left(\omega_{\mathrm{C}}-\omega_{\mathrm{H}}\right)\right. \\
& \left.+3 f\left(\omega_{\mathrm{C}}\right)+6 f\left(\omega_{\mathrm{C}}+\omega_{\mathrm{H}}\right)\right]
\end{aligned}
$$

where $f(\omega)=\left(1+\omega^{2} \tau^{2}\right)^{-1}$, and $\gamma_{\mathrm{C}}$ and $\gamma_{\mathrm{H}}$ are the gyromagnetic ratios for ${ }^{13} \mathrm{C}$ and ${ }^{1} \mathrm{H}, \omega_{\mathrm{C}}$ and $\omega_{\mathrm{H}}$ are the Larmor frequencies for $\mathrm{C}$ and $\mathrm{H}$, respectively, $\hbar$ is Planck's constant divided by $2 \pi, r_{i}$ is the distance between the carbon of interest and the $i$-th hydrogen, and $\tau$ is the single rotational correlation time for the carbon of interest. It must be mentioned here that eq 2 is valid only when the rotational reorientation of the segment is isotropic. Equation 2 may not be strictly applicable to a polymer with a flexible chain, because Shaefer ${ }^{15}$ has shown the existence of a distribution of correlation times for polymers. So the assumption of the isotropic segmental motion may lead to a systematic error. In this paper we use eq 2 as a good approximation.

If the segmental motion (hereafter, this motion is designated as the $\mathrm{R}$ motion) is isotropic in poly(2-vinylpyridine), the $T_{1}$ value of $\mathrm{C}(\alpha)$ must be two times as long as that of $C(\beta)$ according to eq 2 . The experimental results confirm this prediction, and so the $\mathrm{R}$ motion can be characterized by a single correlation time $\tau(\mathbf{R})$.

When there is an internal rotation, the effective correlation time $\tau(\mathrm{I})$ can be described as the sum of two contributions as follows: $:^{16}$

$$
\frac{1}{\tau(\mathrm{I})}=\frac{1}{\tau(\mathrm{R})}+\frac{1}{\tau(\mathrm{G})}
$$

where $\tau(\mathbf{G})$ is the correlation time of internal rotation. The value cannot be calculated directly using eq 2 when the molecular motion is anisotropic due to the contribution of internal rotation. For the calculation of the $\tau(\mathrm{I})$ value, the $T_{1}$ ratio $(1 / \chi)$ is defined by ${ }^{16}$

$$
\frac{1}{\chi}=\frac{T_{1}(\mathrm{I})}{T_{1}(\mathrm{R})}
$$

where $T_{1}(\mathrm{I})$ is the relaxation time when the internal rotation contributes to the relaxation. The $\chi$ value is calculated by the equation ${ }^{16}$

$$
\begin{aligned}
\chi= & \frac{1}{4}\left(3 \cos ^{2} \theta-1\right)^{2}+\frac{18}{5+\rho} \sin ^{2} \theta \cos ^{2} \theta \\
& +\frac{9}{4} \cdot \frac{1}{1+2 \rho} \sin ^{4} \theta
\end{aligned}
$$

where $\theta$ is the angle between the $\mathrm{C}-\mathrm{H}$ vector and the axis of rotation and

$$
\rho=\frac{\tau(\mathbf{R})}{\tau(\mathbf{I})}
$$

As in the case of polystyrene, the internal rotation of poly(2-vinylpyridine) can be divided into two motions: the first is the rotation of the pyridine ring around the $\mathrm{C}(\alpha)-\mathrm{C}(2)$ axis (we call this the $\alpha$ motion) and the second is the precession of the $\mathrm{C}(\alpha)-\mathrm{C}(2)$ in a plane perpendicular to the main chain (we call this the $\beta$ motion). From this model the $T_{1}$ value of $\mathrm{C}(5)$ (which is on the axis of the $\alpha$ motion) is smaller than those of $\mathrm{C}(3), \mathrm{C}(4)$, and $\mathrm{C}(6)$, and larger

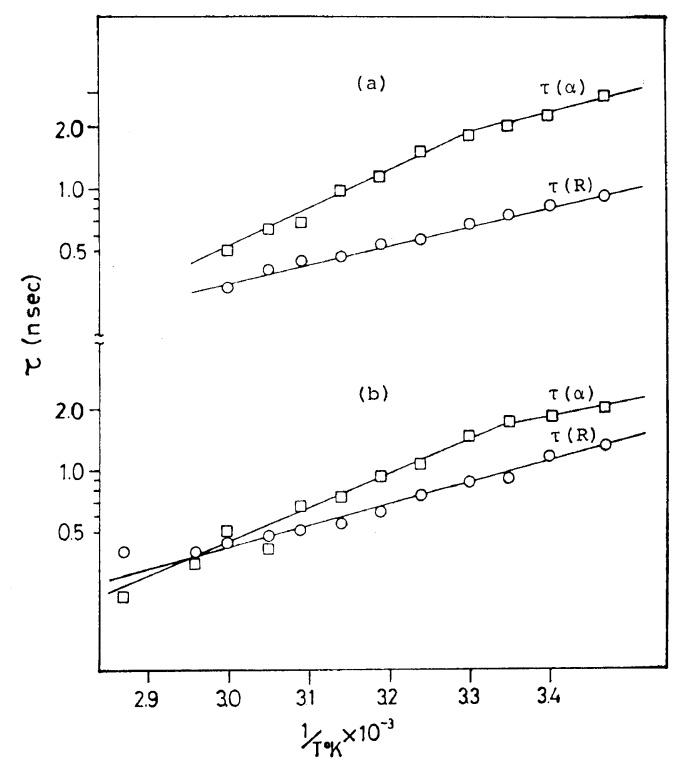

Figure 4. Arrhenius plot of correlation time $(\tau)$ for each molecular motion in poly(2-vinylpyridine): (a) poly(2-vinylpyridine) in chloroform- $d$; (b) poly(2vinylpyridine) in benzene- $d_{6}$. 
than that of $\mathrm{C}(\alpha)$. This is consistent with the experimental results shown in Figure 3, even if a small difference between the factors $\left(\sum r_{i}^{-6}\right)$ for individual carbons is considered. Hence the effective correlation times of individual carbons may be described in terms of the three correlation times of the three elemental motions as follows:

$$
\begin{gathered}
\frac{1}{\tau(\mathrm{C}(5))}=\frac{1}{\tau(\mathrm{R})}+\frac{1}{\tau(\beta)} \\
\frac{1}{\tau(\mathrm{C}(3,4,6))}=\frac{1}{\tau(\mathrm{R})}+\frac{1}{\tau(\alpha)}+\frac{1}{\tau(\beta)}
\end{gathered}
$$

where $\tau(C(5))$ and $\tau(C(3,4,6))$ are effective correlation times of individual numbered carbons, and $\tau(\alpha)$ and $\tau(\beta)$ are correlation times of $\alpha$ and $\beta$-motions, respectively. From eq 4 we can obtain the $T_{1}$ ratio as follows:

$$
\frac{1}{\chi}=\frac{T_{1}(\mathrm{C}(5))}{T_{1}(\mathrm{C}(\alpha))}
$$

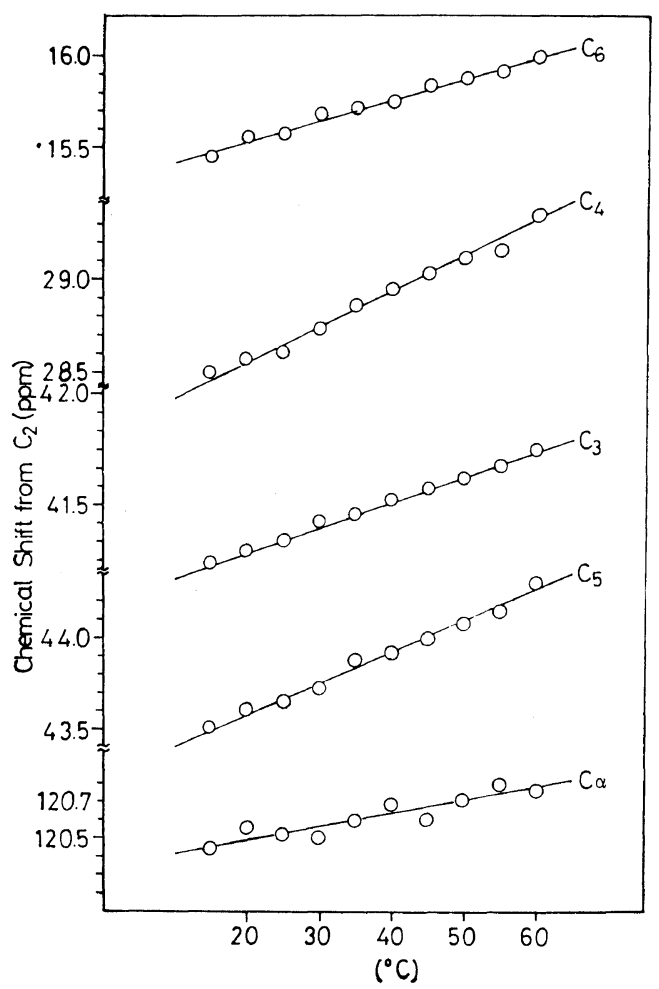

(a) and the relation $1 / \chi\left(90^{\circ}\right)-\rho$ gives the value of $\rho$. Then $\tau(\beta)$ is calculated from the values of $\rho$ and $\tau(\mathrm{R})$ as follows:

$$
\tau(\beta)=\frac{\tau(\mathbf{R})}{\rho-1}
$$

Table I. Activation energy for molecular motion of poly(2-vinylpyridine) (P2VP) in 25\% (w/v) solution and polystyrene (PS) ${ }^{\mathrm{a}}$ in $15 \%(\mathrm{w} / \mathrm{v})$

\begin{tabular}{|c|c|c|c|}
\hline \multirow[b]{2}{*}{ Sample } & \multicolumn{3}{|c|}{$\Delta E, \mathrm{~kJ} / \mathrm{mol}$} \\
\hline & $R$ motion & $\begin{array}{c}\alpha \text { motion } \\
\text { in the } \\
\text { lower } \\
\text { temperature } \\
\text { region }\end{array}$ & $\begin{array}{l}\alpha \text { motion } \\
\text { in the } \\
\text { higher } \\
\text { tempera- } \\
\text { ture region }\end{array}$ \\
\hline $\mathrm{P} 2 \mathrm{VP}$ in $\mathrm{C}_{6} \mathrm{D}_{6}$ & 20.5 & 15.9 & 35.6 \\
\hline $\mathrm{P} 2 \mathrm{VP}$ in $\mathrm{CDCl}_{3}$ & 17.6 & 20.5 & 39.3 \\
\hline $\mathrm{PS}$ in $\mathrm{C}_{6} \mathrm{D}_{5}-\mathrm{CD}_{3}^{\mathrm{a}}$ & 11.7 & 10.5 & 43.9 \\
\hline
\end{tabular}
solution as deduced from Arrhenius plots of the individual correlation times (see Figure 4)

a Cited from ref 6.

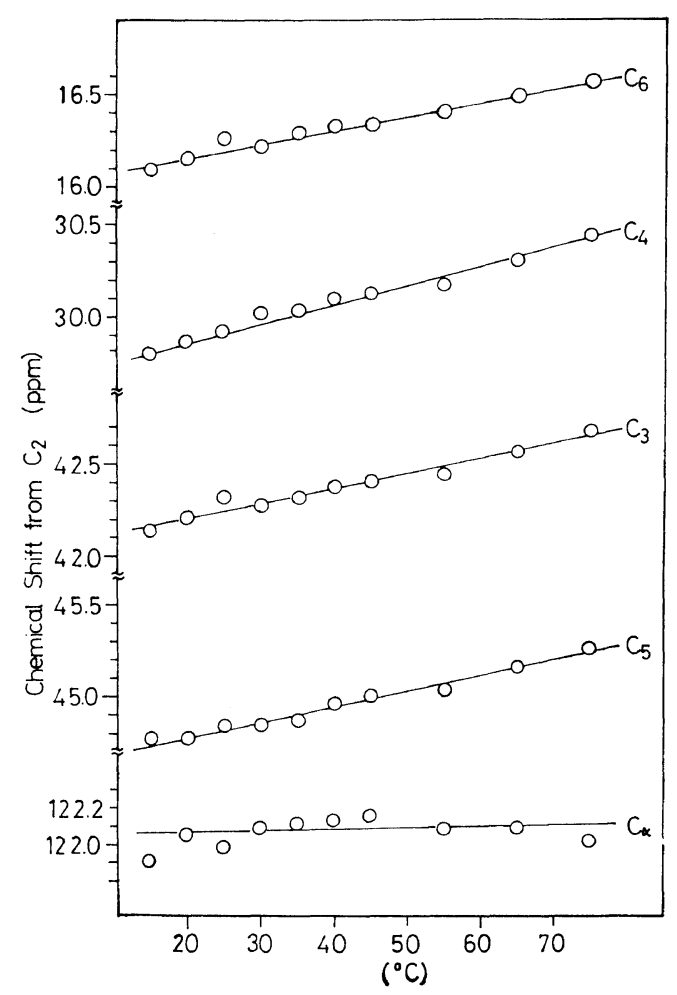

(b)

Figure 5. Temperature dependence of the chemical shift for each carbon in poly(2-vinylpyridine): (a) poly(2-vinylpyridine) in chloroform- $d$; (b) poly(2-vinylpyridine) in benzene- $d_{6}$. The values of chemical shift are expressed in ppm with respect to the $C(2)$ carbon peak. 
In a similar procedure $\tau(\alpha)$ is obtained. The Arrhenius plots of each correlation time are shown in Figure 4.

As shown in Figure 3, the values of $T_{1}(\mathrm{C}(5))$ are very close to those of $T_{1}(\mathrm{C}(\alpha))$, the value of $\rho\left(90^{\circ}\right)$ used for the calculation of $\tau(\beta)$ is very close to unity and so $(\rho-1)$ is very close to zero. This closeness causes the calculated value of $\tau(\beta)$ to include a considerably large error. So in Figure 4 the values of $\tau(\beta)$ are not shown, but we observed linearity in the Arrhenius plot of $\tau(\beta)$ on the whole like that of $\tau(\mathrm{R})$ as shown in Figure 4. Figure 4 indicates that the Arrhenius plots of $\tau(\mathbf{R})$ of poly(2-vinylpyridine) in both $\mathrm{C}_{6} \mathrm{D}_{6}$ and $\mathrm{CDCl}_{3}$ fall on the respective straight lines, while that of $\tau(\alpha)$ shows a break in the vicinity of $25^{\circ} \mathrm{C}$. Thus this transition may be due to the sudden modal change in the rotation of the pyridine ring, as in the case of polystyrene. But the transition temperature is rather lower than that of polystyrene $\left(50-55^{\circ} \mathrm{C}\right)$.

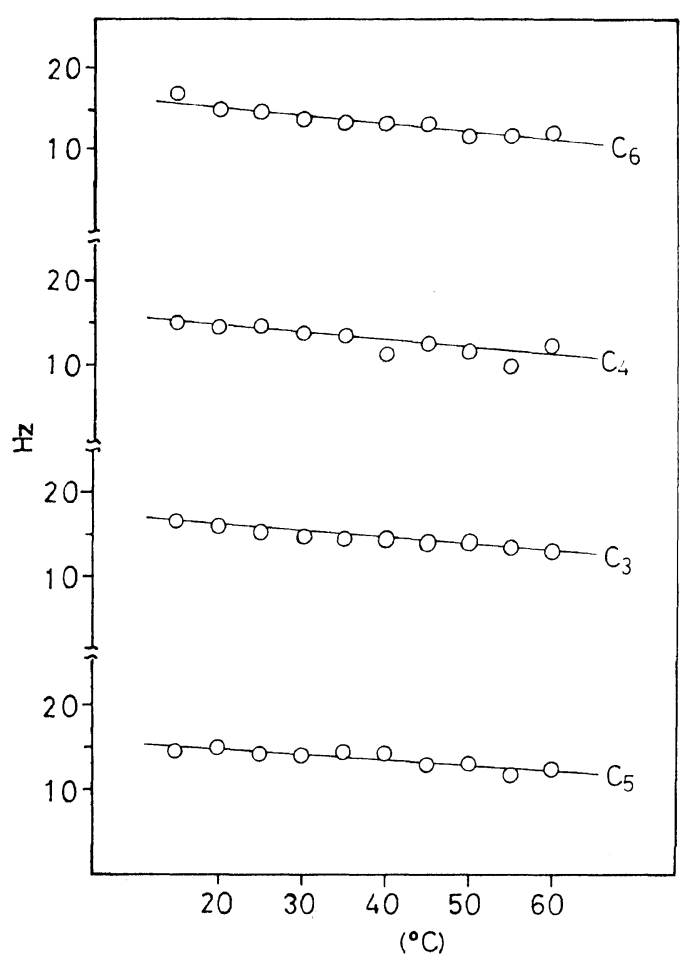

(a)

\section{Activation Energy}

From the Arrhenius plot of the correlation time, the activation energy $(\Delta E)$ for each motion which contributes to the spin-lattice relaxation can be estimated by the equation

$$
\tau=\tau_{0} \exp (\Delta E / R T)
$$

where $\tau_{0}$ is the correlation time expected at infinite temperature and $R$ is the gas constant. In Table $I$ is presented the activation energies of poly(2-vinylpyridine). For comparison the results of polystyrene are also tabulated. The activation energy of each motion of poly(2vinylpyridine) is larger than those of polystyrene except for that of the $\alpha$-motion in the higher temperature region, and the activation energy of the $R$ motion of poly(2-vinylpyridine) in $\mathrm{C}_{6} \mathrm{D}_{6}$ is slightly larger than that of poly(2-vinylpyridine) in $\mathrm{CDCl}_{3}$. On the contrary the activation energy of the $\alpha$ motion of poly(2-vinylpyridine) in $\mathrm{CDCl}_{3}$ is rather larger than that in $C_{6} D_{6}$. This dif-

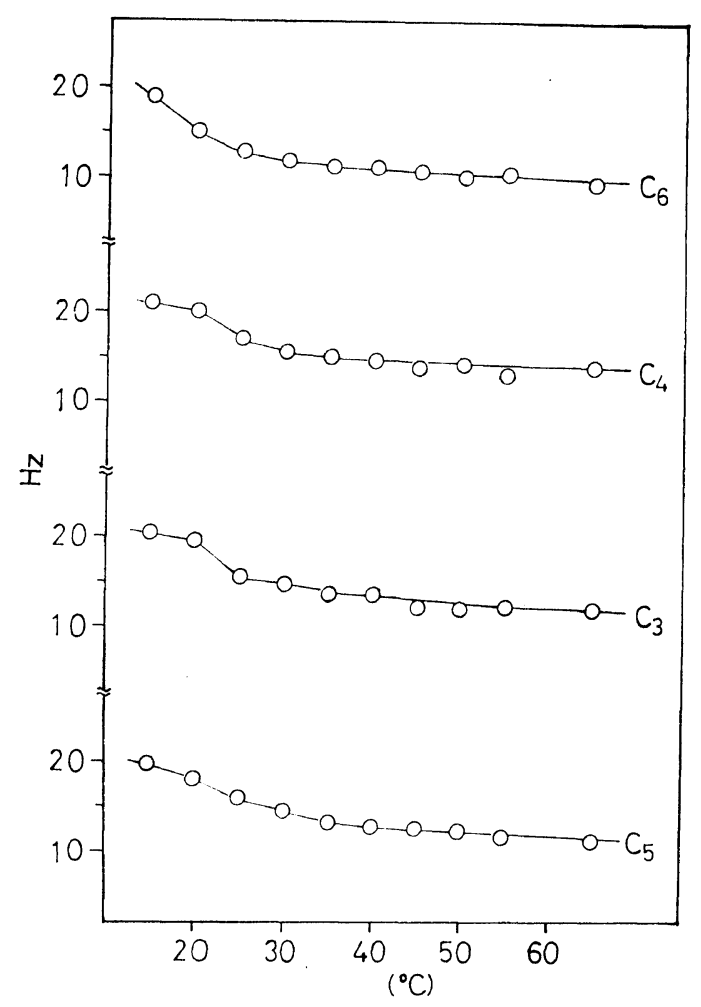

(b)

Figure 6. Temperature dependence of linewidth $\left(\Delta H_{1 / 2}\right)$ deduced from normal spectra: (a) poly(2vinylpyridine) in chloroform- $d$; (b) poly(2-vinylpyridine) in benzene- $d_{6}$. 
ference is assumed to be caused by the difference of the polarity of the solvents. The transition which appears in the temperature dependence of the correlation time of the $\alpha$ motion may be due to the sudden change in the mobility of the $\alpha$ motion, as in the case of polystyrene.

\section{Chemical Shift and Linewidth}

It is very interesting to investigate the influence of the transitional behavior of poly(2-vinylpyridine) in solution which has been discussed above on the chemical shift and linewidth. In Figure 5 is shown the temperature dependence of the chemical shift of poly(2-vinylpyridine) in $\mathrm{C}_{6} \mathrm{D}_{6}$ and $\mathrm{CDCl}_{3}$. The values of chemical shift are expressed in ppm with respect to the $\mathrm{C}(2)$ carbon peak and a positive value corresponds to the upper field. These dependences are linear for both solutions. The $\alpha$ motion of the pyridine ring which has the magnetic anisotropy is expected to influence the temperature dependence of the chemical shift. ${ }^{6}$ However this influence does not appear as a break, but as a linearity in the temperature dependence of the chemical shift, as shown in Figure 5. This may be due to the fact that the $\alpha$ motion at lower temperatures makes the anisotropy of the pyridine ring average out to some extent and the modal change of the $\alpha$ motion at the transition does not influence the temperature dependence of the chemical shift in a step wise way. In Figure 6 is shown the temperature dependence of the linewidth of each peak in carbon-13 spectra of poly(2vinylpyridine) in $\mathrm{C}_{6} \mathrm{D}_{6}$ and $\mathrm{CDCl}_{3}$. In $\mathrm{CDCl}_{3}$, the temperature dependence of the linewidth is linear but in $\mathrm{C}_{6} \mathrm{D}_{6}$, a rapid reduction is observed in the vicinity of $25^{\circ} \mathrm{C}$. This is consistent with the result that the temperature dependence of the intrinsic viscosity of poly(2-vinylpyridine) in benzene shows a transition in the vicinity of $25^{\circ} \mathrm{C}$ but not in chloroform. ${ }^{7}$ This difference may be caused by the difference in polarity of the solvents as discussed below.

\section{DISCUSSION}

Recently Bach Van, et al.,${ }^{17}$ studied the variation as a function of temperature of the UV molar extinction coefficients of dilute solutions of various alkylbenzenes, the configurational isomers of 2,4-diphenylpentane and 2,4,6-tri- phenylheptane, and certain of their halogenated and methylated derivatives. They observed transitional anomalies for these solutions between 30 and $55^{\circ} \mathrm{C}$, and suggested that this temperature range of transition corresponds to a change in the position of the benzene rings with respect to the chain, whether accompanied or not by rotations of the backbone bonds leading to an overall conformational change.

Their results, however, are qualitative and the molecular process occurring in the transition is not clear.

From the analysis of the carbon-13 spin-lattice relaxation time of the poly(2-vinylpyridine) in solution it is concluded that the transition at about $25^{\circ} \mathrm{C}$ is caused by the modal change of the motion of the pyridine ring ( $\alpha$ motion) and not by the segmental motion. At the lower temperatures the internal rotation of the pyridine rings may be restricted by steric hindrance, which is probably due to a repulsive interaction between the hydrogen atoms of the methine group in the backbone and that bonded to the $\mathrm{C}(3)$ carbon in the pyridine ring, ${ }^{6}$ and by the formation of hydrogen bonding between the $\mathrm{N}$ atom in one pyridine ring and $\mathrm{C}(6)-\mathrm{H}$ in another pyridine ring or between the $\mathrm{N}$-atoms in the pyridine ring and chloroform. So the pyridine ring can not rotate freely at the lower temperatures. When the temperature is increased to the higher region, the $\alpha$ motion may be able to overcome the energy barrier due to the steric hindrance and the hydrogen bonding, and the pyridine ring can fully rotate.

The transition temperature of poly(2-vinylpyridine) (about $25^{\circ} \mathrm{C}$ ) is lower than that of polystyrene (about $50^{\circ} \mathrm{C}$ ). ${ }^{1-3,6}$ This difference may be due to the fact that poly(2-vinylpyridine) has only one hydrogen atom which causes the steric hindrance in a pyridine ring but polystyrene has two in its phenyl ring. This interpretation is consistent with the fact that the temperature dependence of optical density of poly(4-vinylpyridine) shows a transition at about the same temperature as polystyrene. ${ }^{8}$ Thus we can say that the main factor which induces the transition is the steric hindrance. The effect of the formation of the hydrogen-bonding on the molecular motion appears in the magnitude of the activation energy. The values of the activa- 
tion energy $(\Delta E)$ of the $\alpha$ motion of poly(2vinylpyridine) in both solutions at the lower temperatures are larger than that of polystyrene in toluene. This result shows that the degree of interruption of the side chain's motion of poly(2-vinylpyridine) is larger than that of polystyrene. The formation of hydrogen bonding reduces the mobility and increases the magnitude of $\Delta E$ of poly(2-vinylpyridine) at lower temperatures. On the contrary, $\Delta E$ of the $\alpha$ motion of polystyrene in toluene in the higher temperature region is larger than those of poly(2-vinylpyridine) in both solutions; the reason for this fact may be the largeness of the steric hindrance of polystyrene compared to that of poly(2-vinylpyridine). It is well known that hydrogen bond formation is disrupted with increasing temperature. ${ }^{18}$ One of the authors has shown that the restriction of molecular motion due to hydrogen bonding drastically decreases with increasing temperature. ${ }^{12}$

It is worthwhile to notice that the values of activation energy of the $\alpha$ motion of poly(2vinylpyridine) in $\mathrm{CDCl}_{3}$ solution are larger than those in $\mathrm{C}_{6} \mathrm{D}_{6}$ solution both in the lower and higher temperature ranges. This result may reflect the different molecular processes occurring in the transition between solutions of $\mathrm{C}_{6} \mathrm{D}_{6}$ and $\mathrm{CDCl}_{3}$. In $\mathrm{CDCl}_{3}$, the nitrogen atom in the pyridine ring may be allowed to make intermolecular hydrogen bonding with hydrogen atoms not only of the pyridine ring but also of the chloroform. ${ }^{7}$ On the other hand, in $\mathrm{C}_{6} \mathrm{D}_{6}$, there is no hydrogen bonding interaction between the polymer and solvent. The formation of the hydrogen bonding between the pyridine ring is not easy because of the steric hindrance. With increasing temperature the hydrogen bondings are weakened and finally broken. The hydrogen bondings once broken at the intermediate temperature range in $\mathrm{C}_{6} \mathrm{D}_{6}$ solution are not readily formed again because the pyridine rings which are fixed to the main chain can not diffuse freely. But in $\mathrm{CDCl}_{3}$ solution a pyridine ring which was bonded to another pyridine ring may reform a hydrogen bonding with the solvent because the solvent molecule can move more freely than the pyridine ring. This is assumed to appear in the data as the differences of the activation energy and the temperature dependences of linewidth and intrinsic viscosity of poly(2-vinylpyridine) in benzene and chloroform solutions.

Acknowledgment. The authors thank Professor R. Chûjô for stimulating discussions during this work.

\section{REFERENCES}

1. G. Moraglio and F. Danusso, Polymer, 4, 445 (1963).

2. C. Reiss and H. Benoit, C.R. Acad. Sci., 256, 268 (1961).

3. G. Weill and R. Reeb, ibid., 263, 21 (1966).

4. C. Reiss, J. Chim. Phys., 1299 (1966).

5. C. Reiss, ibid., 1607 (1966).

6. Y. Inoue and T. Konno, Polym. J., 8, 457 (1976).

7. A. Dondos, Makromol. Chem., 135, 181 (1970).

8. C. Noel and L. Monnerie, J. Chim. Phys., 65, 2089 (1968).

9. C. Chachaty, A. Forchioni, and J. RonfardHaret, Makromol. Chem., 173, 213 (1973).

10. G. Natta, G. Mazzanti, P. Longi, G. Dall'asta, and F. Bernardino, J. Polym. Sci., 51, 487 (1961).

11. S. Arich, Bull. Chem. Soc. Jpn., 39, 439 (1966).

12. Y. Inoue, A. Nishioka, and R. Chûjô, J. Polym. Sci., Polym. Phys. Ed., 11, 2237 (1973).

13. A. Allerhand and R. K. Hailstone, J. Chem. Phys., 56, 3718 (1972).

14. J. Shaefer and D. F. S. Nattush, Macromolecules, 5, 416 (1972).

15. J. Shaefer, ibid., 6, 882 (1973).

16. A. Allerhand, D. Doddrell, and R. Komoroski, J. Chem. Phys., 55, 766 (1971).

17. N.-L. Bach Van, C. Noel, and L. Monnerie, J. Polym. Sci., Symposium, 52, 283 (1975).

18. G. C. Pimentel and A. L. McClellan, "The Hydrogen Bond," W. A. Freeman, San Francisco, California, 1960. 